\title{
UN ABORDAJE HISTÓRICO DE LA ACTIVIDAD FORESTAL EN MISIONES: DEL FRENTE EXTRACTIVO AL AGRONEGOCIO FORESTAL
}

\author{
A historical approach to forest activity in Misiones: \\ from the extractive front to the forestry agribusiness
}

Delia Ramírez*

\section{Resumen}

El presente texto surge de una investigación doctoral extensa e intensa que se realizó en el Alto Paraná misionero, entre los años 2013 y 2016 y se pregunta por las dinámicas -entendidas en términos de relaciones, impactos y consecuencias- del avance del agronegocio forestal en el municipio de Puerto Piray, ubicado en el Alto Paraná misionero, a partir de un trabajo etnográfico realizado en una colonia rural denominada Piray km 18 afectada, específicamente, a las dinámicas del agronegocio forestal. Este artículo tiene el objetivo de realizar un relato histórico del proceso de forestación de la provincia de Misiones, entendiendo que hablar de la historia forestal de Misiones es también referir a la historia de la formación y transformación de los procesos capitalistas en la Argentina.

$<$ Frente extractivo $><$ foresto-industria $><$ agronegocio forestal $>$

\begin{abstract}
The present text arises from an extensive and intense doctoral research that was carried out in the Alto Paraná (Misiones), between 2013 and 2016. It asks about the dynamics - understood in terms of relationships, impacts and consequences - of the progress of the forestry agribusiness in the municipality of Puerto Piray, located in Misiones Alto Paraná. Ethnographic work was carried out in a rural colony called Piray km 18 specifically affected, to the dynamics of the forest agribusiness. This article has the objective of making a historical account of the afforestation process of the Misiones province, understanding that to speak of the forestry history of Misiones is also to refer to the history of the formation and transformation of capitalist processes in Argentina.
\end{abstract}

$<$ Extractive front $><$ forestry-industry $><$ forestry agribusiness $>$

Recibido: 10/09/2017 // Aceptado: 10/12/2017

\footnotetext{
* Licenciada en Comunicación Social (Universidad Nacional de Misiones). Doctora en Antropología Social (PPAS/UNaM). Becaria post doctoral CONICET. Instituto de Estudios Sociales y Humanos (IESyH/CONICET).deliaramirezf@gmail.com
} 
Ramírez. Un abordaje histórico de la actividad forestal en Misiones: del frente extractivo al agronegocio forestal.

\section{Introducción}

El presente artículo aborda los cambios asociados a la producción forestal a partir de una historia de las formas de trabajo y de la organización productiva en la provincia de Misiones. Básicamente esta historiase reconstruye en tres etapas: (i) el frente extractivo: vinculado a la deforestación y la puesta en producción de la tierra con fines mercantiles, que se extiende desde fines del siglo XIX hasta entrado el siglo XX y plantea el escenario adecuado para el proceso de colonización; (ii) el modelo foresto-industrial: relacionado estrechamente con el proceso de industrialización sustitutiva (ISI) que bosqueja un modelo de desarrollo industrial y fabril sobre el que se forjan diferentes configuraciones sociales, que tuvo en la empresa Celulosa Argentina su ejemplo paradigmático; y (iii) el agronegocio forestal, al cual situamos finales de siglo XX respondiendo a un escenario de globalización en el que el capital corporativo establece nuevas formas de organización de la producción, circulación y consumo de las mercancías, así como también nuevas relaciones entre los distintos actores sociales. El agronegocio forestal se basa en la explotación intensiva a gran escala, con procesos de innovación tecnológica y tercerización de actividades y tareas, donde la empresa Arauco encarna por antonomasia el modelo en la región.

De estas tres etapas, enfatizamos en la importancia analítica de la tercera, ya que los cambios asociados al modelo de agricultura globalizada se han acelerado en las últimas décadas y plantean desafíos relacionados con la redefinición de las aproximaciones y herramientas metodológicas en función de comprender aquellas problemáticas que resultan novedosas. En este sentido, enfocamos en la importancia de definir, caracterizar y analizar el agronegocio forestal ya que en Argentina son todavía escasos los trabajos en ciencias sociales que abarcan las problemáticas asociadas a la actividad forestal en el escenario contemporáneo, menos aún desde una perspectiva de la globalización de la agricultura.

Estas reflexiones surgen de una investigación realizada desde la antropología social que apuntó a abordar el tema del agronegocio forestal restituyendo la complejidad de los vínculos entre los actores y los territorios. Sin embargo, la historia, lejos de ser considerada aquí una disciplina "auxiliar" o meramente contextual, cumple un papel muy relevante, en la comprensión de las relaciones entre los actores sociales que interactúan en un territorio acaparado por el agronegocio forestal considerando constantemente las trayectorias y biografías de los actores sociales. En este sentido, la historia vertebra la experiencia de los actores y se reconstruye aquí a partir de indagar la formación de los territorios signados por un paisaje que se caracteriza por la "presencia del pino" y de la "industria forestal".

En este artículo la historia se restituye a partir de las trayectorias individuales y colectivas de los actores sociales. Todas estas cuestiones aparecieron cual "indicios" -en el sentido otorgado por Ginzburg (1994)- en las entrevistas, en los recorridos y visitas a los “pueblos forestales" del Alto Paraná misionero, así como también de las noticias de los medios de comunicación.

Antes de avanzar con el desarrollo del texto, quisiéramos aclarar que la clasificación por etapas se realiza únicamente con fines analíticos y explicativos. Esto 
significa que las etapas no deben pensarse de manera lineal, pues cada una de ellas refiere e incluyen diferentes movimientos -incluso contradictorios-, ni tampoco deben pensarse a modo evolucionista y unidireccional, pues las formas y modalidades productivas de explotación y producción forestal han convivido en los territorios a lo largo de la historia. Hablar de un "frente extractivo", de la "foresto-industria" o del "agronegocio forestal" alude a los modelos que primaron en la economía y en la política, con sus correlatos de producción simbólica en cuanto de explotación y producción del recurso que en las diferentes épocas.

Asimismo, es preciso aclarar que no nos importan solo las modalidades de producción; no es nuestra intención realizar una "historia del trabajo forestal", sino explorar las formaciones y configuraciones sociales que se han gestado a partir de la implementación de los diferentes modelos de producción.

Finalmente, aclaramos que aun cuando este artículo reconstruye un proceso observado en la provincia de Misiones, no es nuestro objetivo realizar un abordaje "regionalista" del proceso, sino pensar la articulación constante entre los cambios en la agricultura a nivel local, nacional y mundial, en el marco de un modelo de acumulación dispuesto por una economía global. Misiones es una de las más importantes provincias a nivel nacional en lo que a la actividad forestal respecta. Hablar de la historia forestal de Misiones es también referir a la historia de la formación y transformación de los procesos capitalistas en la Argentina.

\section{El frente extractivo}

En el siguiente apartado recurrimos al concepto "frente extractivo", postulado por Abínzano (1985) en un trabajo clásico de la antropología social, quien lo definió como la forma predominante de ocupación y organización de los circuitos económicos de la época siglos (XIX y principios del XX). También hemos revisado otros trabajos historiográficos y antropológicos (Scalerandi, 2012; Kraustofl, 1912; Alcaráz, 2013; Bartolomé 2007, entre otros) a los que haremos referencia. Recuperar el concepto de frente extractivo postulado por Abínzano permite comprender que la actividad forestal estuvo asociada desde los tiempos de la colonización, e incluso antes, a las prácticas de deforestación. De esta manera discutimos con visiones instaladas que piensan a la actividad forestal únicamente en términos de industrias que se generan alrededor de las plantaciones de rápido crecimiento.

El frente extractivo implica un modelo de ocupación y utilización del suelo y, simultáneamente, un sistema productivo de baja inversión; destrucción de recursos no renovables a corto plazo y su inserción en un sistema de mercado regulado desde fuera de la propia región por los sectores comerciantes ubicados en el puerto de Buenos Aires, que pronto desarrollaron el monopolio de la industrialización definitiva del producto y su comercialización masiva. Abínzano abordó la constitución de una sociedad multiétnica en una región de frontera y desde esa mirada reconstruyó el espacio social dentro de dinámicas regionales de producción. 
Ramírez. Un abordaje histórico de la actividad forestal en Misiones: del frente extractivo al agronegocio forestal.

La actividad forestal en aquella época implica, por un lado, extraer recursos de los yerbales naturales y deforestar el monte para hacer uso de la madera. Los trabajadores eran los mensúes, llamados así porque su paga era mensual. La mayoría de ellos eran mestizos, criollos e indígenas provenientes sobre todo del Paraguay, aunque también de otras provincias argentinas como Corrientes y Entre Ríos. También estaban los hacheros, así denominados por el uso del hacha como herramienta; trabajaban en la selva en condiciones de baja salubridad y con altos riesgos en las operaciones forestales (Abínzano, 1985).

Las disposiciones de creación del Territorio Nacional de Misiones del presidente Julio A. Roca en diciembre de 1981 favorecieron a la recepción de numerosos contingentes de inmigrantes extranjeros. Se inició entonces un proceso de consolidación de la agricultura que adquirió mayor intensidad después de la década de 1920 cuando los yerbales naturales llegaron a su límite económico y entonces se produjo la expansión de los yerbales implantados. Así, la provincia de Misiones devino en la principal productora de este cultivo, y el colono pasó a ser el actor social responsable de transformar las fronteras naturales en territorios productivos.

Durante la mayor parte del siglo XX, se consideró a la selva como un freno al progreso, un espacio sobre el cual se debía avanzar (Ferrero, 2006). Pero en la década de 1930 se introducen cambios institucionales - con la creación de la Sección Técnica de Bosques dentro de la Dirección de Tierras, dependiente ésta del Ministerio de Agricultura de la Nación con el asesoramiento técnico de especialistas forestales extranjeros ${ }^{1}$ - que influye en la organización productiva de la actividad forestal en el nivel territorial ya que se apuntala de manera incipiente una industria local. A finales de esa década, en Misiones se apuntó a producir madera aserrada y otros productos derivados, todavía rústicos. Alrededor de la elaboración de esos productos se instalaron las fábricas y las villas obreras (Scalerandi, 2012), conformadas por obreros que trabajaban en esas primeras industrias. Kraustofl (1991) hace hincapié en el sector dedicado a la extracción de madera del bosque nativo, atendiendo particularmente a la organización del sistema productivo, las condiciones específicas de trabajo y calidad de vida de los peones que participan en dicho proceso.

En consecuencia es posible hablar de un segundo momento de este frente extractivo, en el cual surgieron industrias muy rudimentarias (molinos, obrajes, aserraderos, etcétera) que seguían ligadas a la explotación de especies nativas.

En principio, estuvieron los obrajes generalmente cerca de los ríos ya que se utilizaban los cursos de agua para transportar los rollos de madera. Los obrajes posicionaron una elite local, cuyo poder político y económico se consolidó con la explotación yerbatera-maderera a partir de los grandes latifundios establecidos desde el Gobierno Nacional. En este sentido, esta elite forjó su podera partir de alianzas estratégicas con las elites centrales de las grandes ciudades (Alcaráz, 2013). Posteriormente, los obrajes y campamentos de obrajes fueron declinando con la degradación del monte (Kraustofl, 1991; Scalerandi, 2012).

\footnotetext{
1 Ver http://www.agroindustria.gob.ar/new/0-0/forestacion/_archivos/_biblioteca/ifona22.htm
} 
Luego se instalaron en el monte pequeñas y medianas industrias que dieron continuidad a los denominados campamentos de obraje que se ocupaban de la extracción de rollos. Alrededor de dichos campamentos se generaron las villas obreras donde vivían los trabajadores de los aserraderos, laminadoras y fábricas de terciado así como otros trabajadores, que sin ser asalariados permanentes de la industria, contratados de manera transitoria para las tareas de limpieza y plantación (Scalerandi, 2012).

El paso de un modelo puramente extractivista a uno con procesos básicos de industrialización realizados localmente -y consecuentemente el desplazamiento del obraje como centro de la organización socio-productivo a la fábrica- se inscribe en el marco más amplio del cambio a nivel nacional del modelo agro-exportador al modelo de industrialización por sustitución de importaciones (ISI) que, en el caso de la madera, tuvo por objetivo la provisión de productos terminados e insumos intermedios al mercado interno en base al aprovechamiento de los recursos forestales. Este proceso representa la antesala de la etapa a la que llamamos industrialista, industrial-forestal o de la foresto-industria, la cual abordaremos en el siguiente apartado.

\section{El modelo forestal-industrial}

Esta etapa que clasificamos como "industrialista", del modelo industrial forestal" o de la "foresto-industria" se extiende desde mediados del siglo XX hasta finales de la década de 1980 y se inscribe en la perspectiva desarrollista del Estado, que consideraba primordial una industrialización del agro para el crecimiento de los territorios locales. En ese contexto, se produjo en el Alto Paraná misionero un continuo y persistente crecimiento de las plantaciones forestales con destino industrial que modificó sustancialmente el paisaje del norte de la provincia.

El impulso a la instalación de las plantas industriales de celulosa en Misiones se debió no sólo a las condiciones ecológicas de la región que propiciaban el rápido crecimiento de las especies implantadas, materia prima para las fábricas de celulosa, sino que también fue parte de las nociones de desarrollo propias de aquel tiempo: la actividad forestal se presentó como un modelo más industrial que agropecuario, ya que se consideraba que la industria generaba mayor valor agregado, por ende mayor empleo, por ello se apostaba a los proyectos industriales como motor del desarrollo.

La consolidación del modelo de la foresto-industria se caracteriza por el establecimiento de industrias papeleras en la provincia de Misiones, el crecimiento de las plantaciones con fines industriales y la formación de pueblos y colonia alrededor aserraderos y emprendimientos fabriles. La tecnología empleada en las nuevas fábricas superaba a las rudimentarias del modelo extractivista; así el Alto Paraná misionero comenzó a considerarse como un polo foresto-industrial.

La Dirección Forestal del Ministerio de Agricultura (1943) representó la primera manifestación o reconocimiento de la importancia del recurso forestal dentro de la economía nacional (Mastrangelo et al., 2011). El modelo de la foresto- industria ha sido promovido con la promulgación de la Ley Nacional de Defensa de la Riqueza forestal 
Ramírez. Un abordaje histórico de la actividad forestal en Misiones: del frente extractivo al agronegocio forestal.

$\left(\mathrm{N}^{\circ} 13.273\right)$. Por medio de esta Ley se creó la Administración Nacional de Bosques (ANB), como organismo de aplicación; "como parte de los planes de explotación esta ley promovía, mediante exenciones impositivas a la inversión, la elaboración in situ del recurso natural para generar puestos de trabajo y agregar valor localmente" (Mastrangelo et al., 2011: 101).

Más tarde, la aparición de la Celulosa Argentina en el municipio de Puerto Piray representaba la propuesta de un modelo de desarrollo basado en la fábrica, empleando obreros en las zonas rurales y urbanas e impactando en el desarrollo de barrios y colonias. Puerto Piray, pasó de ser un puerto de carga y descarga de recursos extractivos (madera y yerba) a un pueblo fabril con miles de habitantes a partir de la instalación de "la Celulosa", como la llaman los habitantes de Puerto Piray.

La firma Celulosa Argentina llegó al municipio de Puerto Piray en 1942, en 1950 comenzó la construcción de la fábrica, la cual se concluyó un año después. Con la inauguración de la fábrica en 1956 se transformó no solo el municipio, que hasta ese momento era un puerto en el que vivían unos pocos habitantes, sino la forma de producción, el destino principal de las plantaciones forestales, y la organización rural y urbana planteada en torno a la misma. Desde el puerto se avanzaba cortando árboles nativos, preparando los terrenos y luego reforestando con árboles que servirían de insumo para la fabricación de celulosa. Entonces se armaban campamentos y luego viviendas para los obreros rurales que se instalaban con sus familias.

La condición de frontera de Puerto Piray favoreció a la recepción de migrantes paraguayos que venían a trabajar y a vivir en la Argentina. Puerto Piray se convirtió en uno de los lugares de atracción de la población migrante pues la pujante fábrica ofrecía oportunidades de obtener empleo. La población era empleada en las tareas de desmonte, forestación y luego en la fábrica.

Puerto Piray se organizó en torno a los barrios próximos a la fábrica, que hoy son parte del área urbana del municipio, y pequeñas colonias rurales ubicadas en las zonas de las forestaciones. Muchos de estos lugares son conocidos hasta la actualidad, muchos desaparecieron, como los kilómetros (Km. 18, Km. 22, Km. 28, Km. 36) que eran tierras productivas de Celulosa Argentina. En los kilómetros habitaban los obreros que abrían las picadas y luego trabajaban en las plantaciones forestales (fundamentalmente de Pino Paraná, una especie nativa, Araucaria angustifolia). También se instalaron muchos obreros que trabajaron para una fábrica de resina que ya existía en Puerto Piray, ya que la resina se obtenía de estos mismos pinos. Posteriormente, esta fábrica desapareció con el cierre de Celulosa Argentina.

Antes del surgimiento de otras fábricas de celulosa como Papel Misionero (1975) y Alto Paraná (1982), Celulosa Argentina fue la fuente laboral más importante que tenía Puerto Piray, e incluso la provincia. Varios de los entrevistados insistieron en lo importante que fue la Celulosa Argentina para el pueblo y para sus vidas personales: "Celulosa era palabra mayor, hasta la ciudad de Eldorado dependían casi todos de la Celulosa. En el '80 terminó todo” (vecino de Piray km 18, entrevistado el 16 de febrero de 2016). Según un ex empleado de Celulosa Argentina, en su momento de mayor 
esplendor, trabajaban en esta empresa aproximadamente 700 personas, la mayoría de ellas vivían en Puerto Piray.

La fábrica Celulosa Argentina funcionó normalmente hasta 1980, pero luego entró en un período de crisis y pérdidas recurrentes y fue cerrando progresivamente las diferentes sucursales que tenía a lo largo del país. En sus últimos años, la empresa Celulosa Argentina había pasado a tercerizar parte de las tareas que antes llevaba a cabo mediante la contratación directa de asalariados: "En ese tiempo la empresa era Celulosa, después cambió el patrón. En ese tiempo había todavía asegurado gente, cantidad de gente y nosotros somos los changarines trabajamos con contratistas, pero todo para Celulosa" (vecino de Piray km 18, entrevistado el 20 de febrero de 2016). Según manifestaron ex obreros que trabajaron para Celulosa o para contratistas de la misma, la crisis se sintió especialmente entre los trabajadores de menores salarios, que fueron despedidos, en muchos casos sin ser correctamente indemnizados.

Celulosa Argentina y la creación en 1975de la empresa estatal Papel Misionero (colonia Puerto Mineral, municipio Puerto Leoni, departamento Libertador General San Martín) promovieron la tecnificación tanto a nivel silvicultural, es decir, del manejo de las plantaciones forestales desde la reforestación hasta la cosecha, como industrial, en la transformación de la madera proveniente de las plantaciones, tanto en fábricas que se radicaron sobre la costa del río Paraná como en el distrito industrial en la Ciudad de Eldorado (Scalerandi, 2013).

El salto cualitativo en la constitución del modelo foresto-industrial se produjo en la década de 1970 con el fortalecimiento de los organismos estatales de apoyo a la producción forestal y la creación de leyes de promoción de esta actividad ${ }^{2}$. Como resultado de estos programas de promoción, varias empresas grandes y medianas, todas de capitales nacionales, se instalaron en Misiones para dedicarse a la forestación. La desgravación impositiva del año 1972 incentivó a que los valores consignados como utilidad en las declaraciones juradas del impuesto a los réditos se volcaran al proceso forestal. En el año 1973 se creó el Instituto Forestal Nacional (IFONA) en el marco del Ministerio de Economía, como un organismo autárquico del Estado, con funcionamiento ajustado a las directivas del Poder Ejecutivo para el fomento de la forestación. Su objetivo fundamental fue lograr el mayor abastecimiento interno de maderas, pastas celulósicas, papeles y demás productos forestales, mediante el aprovechamiento equilibrado de los bosques nativos, incremento en obras de forestación con especies de rápido crecimiento y radicación de actividades transformadoras, todo ello con resguardo del medio ecológico y bienestar general del país ${ }^{3}$. En 1974 se sancionó el decreto

\footnotetext{
2 La ANB (Administración Nacional de Bosques) llegó a contar con 1200 agentes entre técnicos, administrativos y personal de apoyo. Organizó actividades y congresos importantes en la década de 1950. En el año 1968 se convierte en Servicio Nacional Forestal y en 1969, como consecuencia de una nueva estructura orgánica de la Secretaría de Estado de Agricultura y Ganadería pasa a denominarse Servicio Nacional Forestal, con la misión de "Entender en la ejecución y fomento de los recursos forestales naturales, y asi como también en la ejecución de los programas de investigación... ”http:// www.agroindustria.gob.ar/new/0-0/forestacion/_archivos/_biblioteca/ifona22.htm

3 La ANB (Administración Nacional de Bosques) llegó a contar con 1200 agentes entre técnicos,
} 
Ramírez. Un abordaje histórico de la actividad forestal en Misiones: del frente extractivo al agronegocio forestal.

465/74 de fomento a la forestación basada en especies exóticas, así como también en desgravaciones impositivas que cubrían un alto porcentaje de la inversión.

En la década de 1980 se sancionó la ley de desgravación fiscal para las tierras de baja productividad que apuntó a incrementar la producción. La misma estableció una mecánica de recuperación de terrenos para ser utilizados en la producción agropecuaria. En esta ley se definía como tierras de baja productividad las áridas, anegadizas, con problemas de salinidad y superficies boscosas (Valls, 2000). La ley se aplicó hasta 1987 y los beneficiarios fueron principalmente grandes contribuyentes del impuesto a las ganancias y no alcanzó prácticamente a pequeños productores.

Como se observa, el monte seguía pensándose como la antítesis o el freno al desarrollo. Ferrero (2006) contrasta varios estudios y determina que la reducción de bosque nativo entre los años 1950 y 1977 fue de un 53\%. Esto coincide con afirmaciones de Gómez Lende (2016), que sostiene que si bien el área forestada con especies exóticas (pinos, eucaliptos, kiri, paraíso) creció rápidamente en Misiones, Corrientes, Entre Ríos y Buenos Aires, el $90 \%$ de la madera procesada por la industria continuaba proviniendo del bosque nativo.

A mediados de la década de 1980, se estancaron los tiempos de bonanzas para el sector de la foresto-industria, cuando una fuerte crisis económica a nivel nacional puso fin a los créditos fiscales para la forestación (Gómez Lende, 2016). Esta situación afectó a Celulosa Argentina y finalmente la empresa se declaró en quiebra a finales de la década de 1980. Al contexto de crisis económica que atravesaba el país durante esos años, que derivarían en la hiperinflación de 1989 y la salida anticipada del gobierno de Raúl Alfonsín -que la empresa Celulosa Argentina no pudo sortear- se sumaba la desinversión por parte de los empresarios del grupo propietario.

La propiedad de esta empresa pasó a manos de Celulosa Puerto Piray (CPP) que tenía un proyecto de construcción de una nueva fábrica de celulosa que nunca llegó a concretarse. Celulosa Argentina continuaba siendo accionista en ese proyecto hasta que entró en quiebra y pasó a manos del Citibank a principios de la década de 1990 y luego pasó a manos de la empresa ARAUCO, que adquirió también las tierras de las plantaciones forestales que Celulosa poseía en el municipio de Puerto Piray.

El quiebre de la Celulosa Argentina representa el declive de la etapa industrialista y el arribo de la multinacional ARAUCO se vincula a la emergencia del modelo del agronegocio forestal como el nuevo esquema que organiza las relaciones productivas, sociales y políticas. Desarrollaremos esta cuestión en el siguiente apartado.

administrativos y personal de apoyo. Organizó actividades y congresos importantes en la década de 1950. En el año 1968 se convierte en Servicio Nacional Forestal y en 1969, como consecuencia de una nueva estructura orgánica de la Secretaría de Estado de Agricultura y Ganadería pasa a denominarse Servicio Nacional Forestal, con la misión de "Entender en la ejecución y fomento de los recursos forestales naturales, y asi como también en la ejecución de los programas de investigación... "http:// www.agroindustria.gob.ar/new/0-0/forestacion/_archivos/_biblioteca/ifona22.htm 


\section{El agro-negocio forestal}

Los conceptos de agro-negocio y agro-negocio forestal se utilizan aquí como categorías analíticas que dan cuenta de un nuevo modelo productivo basado en la producción a gran escala, el capital intensivo, las nuevas formas de organización de la producción, así como de acceso y explotación de los distintos recursos naturales.

El modelo agribusiness o del agronegocio (Gras y Hernández, 2014) se sustenta en intensos cambios tecnológicos, nuevas formas de acumulación (ya no es la industria la mayor captadora de excedentes sino las empresas de insumos) y la concentración productiva. En líneas generales, se caracteriza por la centralidad del papel del capital y su conexión con la economía globalizada. Se trata de un tipo de agricultura de gran escala, que no puede comprenderse por fuera del proceso de globalización capitalista y cuyo sistema está conformado por un conjunto de prácticas, relaciones y representaciones vinculadas a una visión sobre los "modos de hacer negocio" en la agricultura.

En este último apartado, enfatizamos el papel de la empresa multinacional ARAUCO S.A; que se instala con fuerza en Misiones a mediados de la década de 1990 y produce cambios en las modalidades y esquemas productivos, transformaciones institucionales vinculadas a las intervenciones estatales y nuevas configuraciones sociales que implicaron, en muchos casos, la desaparición de poblados completos cuyas tierras pasaron a destinarse a plantaciones.

La aparición del agronegocio se produce en un contexto capitalista de globalización de la agricultura, en la década de 1990. Se desarrolló en Argentina mediante una serie de cambios institucionales, tecnológicos, productivos y financieros, que afianzaron la producción agroforestal en pocos cultivos exportables. Los efectos de estas transformaciones sobre la estructura social fueron determinantes. La nueva dinámica de acumulación se basó en un proceso de concentración empresarial, el desplazamiento de la agricultura familiar y el arrinconamiento del campesinado.

El agronegocio supone una lógica potente en términos de la subordinación de la agricultura al capital agro-industrial. Ello se observa en el control que el mismo adquiere sobre el material genético, a través de la biotecnología y su asociación a paquetes específicos de agroquímicos. En esa línea también, en lo referido a los sistemas de innovación, se conforman paisajes institucionales controlados por actores privados, que desplazan a los organismos públicos de la investigación y el desarrollo de tecnologías agropecuarias (Gras, 2013). Asimismo, el agro-negocio se caracteriza por el desarrollo de pocas actividades agropecuarias en los territorios en los que se inserta, es decir, implica una tendencia al monocultivo que se expresa en el aumento sostenido de la superficie cultivada y el desplazamiento de otros usos del suelo (Gras, 2013).

La soja es el cultivo emblemático en Argentina al igual que en el resto del Mercosur, por la relevancia que ha tomado en función de su exportación como alimento para el ganado de Europa y China.

Por la forma en la que se desarrolló la actividad forestal en Misiones desde la década de 2000, es posible determinar la modalidad del agro-negocio forestal, pues 
Ramírez. Un abordaje histórico de la actividad forestal en Misiones: del frente extractivo al agronegocio forestal.

sus lógicas de producción y comercialización dan cuenta de las características ya mencionadas, como: (i) la inserción de la producción en una cadena global de valor; (ii) el creciente peso de la producción de "commodities" frente a la de productos con mayor valor agregado; (iii) el interés de grandes grupos agrarios y no agrarios, en especial capitales financieros, por los recursos forestales y su creciente capacidad para orientar las inversiones y lógicas productivas a las necesidades de abastecimiento de las cadenas globales; (iv) la producción a gran escala, tanto en tierras como en capital; (v) la centralidad del actor empresarial, quien opera en base al conocimiento del negocio agrícola global y lógicas basadas en el sistema financiero (es decir, la búsqueda de una rentabilidad semejante a la del negocio financiero); (vi) el desplazamiento de actores tradicionales, en particular los productores de la materia prima; y (vii) la constante innovación tecnológica que genera un campo de investigaciones científicas a su servicio.

El agronegocio forestal responde a un modelo globalizado, es decir, se encuentra inserto en Cadenas Globales de Valor $(\mathrm{CGV})^{4}$. Esta orientación hacia el mercado internacional y hacia consumidores globales determina las formas de explotación y gestión de los recursos, los patrones de inversión, las modalidades de innovación tecnológica y la organización de los procesos de producción y trabajo. Estos elementos tienen consecuencias en las estructuras sociales preexistentes, y se materializan en los escenarios locales, reconfigurando relaciones sociales, económicas y políticas. En este contexto, adquieren centralidad los actores empresariales que ponen en juego nuevos modos de relación con el resto de los actores (Estado, trabajadores, otros productores, población local, etc.).

De la producción de árboles se obtienen dos subproductos principales: la madera y la pasta celulósica); ambos son commodities-esto es, bienes con bajo nivel de diferenciación y valor agregado- que a su vez son demandados para otros procesos industriales vinculados a su transformación física o química. Recientemente se ha incluido la producción de energía a través de procesos termoquímicos como parte del agronegocio forestal. Tanto la madera como la pasta celulósica tienen exigencias específicas en cuanto a los insumos, la fuerza de trabajo, la maquinaria y la tecnología que requieren.

En Misiones, las especies de rápido crecimiento que predominan son el Pinus Elliotis y el Taeda. Asimismo, es importante mencionar que la actividad forestal se sirve de una constante innovación tecnológica propia del modelo productivo del agronegocio a partir de un apoyo constante a la investigación científica de la cual se sirven y se nutren las empresas del sector. El empresario forestal moderno opera en base al conocimiento

\footnotetext{
${ }^{4}$ Las cadenas globales de producción o cadenas globales de valor (CGV) son sistemas asociados al proceso de globalización. Los avances en las tecnologías de la información y las comunicaciones, los menores costos de transporte, la liberalización del comercio y el auge de la inversión extranjera directa promueven una mayor conexión e interdependencia de empresas transnacionales que anteriormente operaban geográficamente dispersas, integrándolas a un circuito de producción y distribución (Bianchi y Szpak, 2013). En Uruguay (Cerdeillac y Piñeiro, 2016; Carámbula, 2016) se investigan las relaciones sociales que se forjan con el agronegocio en el marco de CGV. Los estudios uruguayos fueron constantemente revisados durante mi investigación por la importancia que la expansión forestal tiene para ese país y para la agenda académica en los últimos años.
} 
introduce nuevas lógicas de gestión y comercialización en función de las relaciones de integración global del modelo productivo. La producción se basa, como ya fuera mencionado, principalmente en la gran escala tanto en términos de superficie como en el uso intensivo del capital.

En síntesis, en Misiones el modelo del agronegocio no ingresó con el cultivo de soja sino con las plantaciones forestales de rápido crecimiento bajo modalidades productivas de manejo empresarial perfiladas globalmente. Sin dudas, el tipo de cultivo influye en las dinámicas ya que el agronegocio sojero no es idéntico al forestal. Las formas y características de explotación del tipo de cultivo y la importancia de su renta a nivel nacional imprimen especificidades.

En lo que respecta a los movimientos económicos y empresariales, la actividad forestal de Misiones tiene reducido peso en el total de las exportaciones nacionales (aproximadamente el $2 \%$ ), pero es de relevancia en la estrategia oficial de desarrollo promovida desde el Estado provincial y el sector empresarial, que destacan su importancia para la economía provincial. No obstante, el análisis de importacionesexportaciones del sector forestal de los últimos años muestra que la balanza comercial mantiene un alto déficit determinado por la importación ${ }^{5}$.

Las medidas político-institucionales fomentadas por el Estado Nacional durante la década de 1990 forjaron las condiciones para el ingreso de capitales extranjeros a la actividad. Según Gómez Lende (2016), la inversión extranjera directa que el complejo foresto-industrial argentino recibió entre 1990 y 2000 ascendió a 3.500 millones de dólares, en su mayoría provenientes de Chile (46\%), Estados Unidos (31\%), Canadá (15\%), y en menor medida desde Inglaterra, Brasil, Holanda, Alemania, Nueva Zelanda, Irlanda, España, Corea del Sur, Italia y Uruguay. Incluso agentes financieros globales de los granos (Louis Dreyfus) y el petróleo (Shell) y hasta un fondo de pensión de docentes de la Universidad de Harvard se sumaron al auge forestal argentino. Esas inversiones se concentraron sobre todo en las provincias de Misiones (40\%) y Corrientes (20\%), y en menor medida, en Entre Ríos, Neuquén, Córdoba, Buenos Aires y Salta.

El Gobierno Nacional cumplió un papel importante en la rápida expansión de la superficie forestal en la provincia. Si bien a comienzos de la década de 1990, se disolvió el IFONA, en 1998, se aprobó la ley 25.080 de "Inversiones para bosques cultivados", la cual fue prorrogada en el 2008 hasta el 2018. La misma estableció un régimen de promoción de las inversiones para el fomento de emprendimientos forestales con el objeto de beneficiar la instalación de nuevos proyectos forestoindustriales y la ampliación de los existentes. A ello se sumaron los fondos otorgados por el Consejo Federal de Inversiones (CFI), el área de Desarrollo Regional de la Subsecretaría de la Pequeña y Mediana Empresa (FONAP y ME), el Programa de Apoyo a la Restructuración Empresarial (PRE). Estos programas fomentaron la actividad de silvicultura y permitieron la expansión de la forestación fundamentalmente en el Alto Paraná misionero. Asimismo, se puede mencionar la extensión de tierras destinadas a la

\footnotetext{
5 Ver informe Evolución del comercio internacional de productos forestales 2010-2013. Peirano, C. (2014),
} Asociación Forestal Argentina (AFOA), Abril 2014. 
Ramírez. Un abordaje histórico de la actividad forestal en Misiones: del frente extractivo al agronegocio forestal.

forestación bajo el régimen de "Promoción de plantaciones forestales" en la década de 1990 y que afectó principalmente a Misiones y Corrientes (Slutzky, 2014).

En paralelo a la implementación de estas lógicas en Misiones, en pocos años, se produjo un incremento de las plantaciones forestales a un ritmo vertiginoso: en el año 1992, la superficie cultivada con bosques implantados era de 7.347 hectáreas, mientras que en 1999 alcanzaba las 50.000 hectáreas, y para el año 2004 aumentó a 240 mil hectáreas (Ferrero, 2003). A partir de 2010, Misiones cuenta con más de 370.000 hectáreas de plantaciones forestales y se dice que el crecimiento de las especies forestales en esta provincia es casi el doble comparados con los países de tradición forestal ${ }^{6}$. En contraste, durante el mismo período la caída de los precios de los tradicionales cultivos industriales de la provincia (tung, te, yerba), el incremento de costos generados por el régimen de convertibilidad, vigente entre 1991 y 2002 (que estableció la paridad entre el peso argentino y el dólar), y la importación de productos alimenticios desde Brasil, destruyeron las capacidades de producción de los agricultores familiares (Chifarelli, 2010).

A finales del Siglo XX, la Ley de Bosques Cultivados y la Ley de Estabilidad Fiscal (ley 25.080 de 1998 prorrogada en 2008) otorgaron nuevos beneficios al sector forestal: (i) exención fiscal de impuestos nacionales (inmobiliario, sellos, ingresos brutos, ganancia mínima presunta y patrimonio); (ii) amortización acelerada del Impuesto a las Ganancias; (iii) devolución del impuesto al valor agregado; (iv) desgravación arancelaria a las importaciones de insumos y maquinarias; (v) congelamiento de tasas durante 30 años; y (v) aportes estatales para forestación, discriminados según extensión, especies, actividades, modalidades de explotación y provincias (Gómez Lende, 2016).

Misiones se convirtió en una de las principales provincias forestales del país, por el peso relativo de las plantaciones: concentra el $25 \%$ de la superficie total de bosques implantados a nivel nacional, seguida por Corrientes y Entre Ríos (Ferrero, 2006). Más del $60 \%$ de las plantaciones forestales de Misiones se encuentran en los departamentos que están sobre el río Paraná.

\section{La gran escala en la explotación de la tierra: dimensión fundamental en la caracterización del agronegocio forestal}

La necesidad de contar con grandes extensiones de tierra ${ }^{7}$, lo que denominamos gran escala, se relaciona con la necesidad de las empresas de contar con un volumen de producción anual suficiente para asegurar su rentabilidad. El ciclo productivo del cultivo de árboles demora entre 7 a 15 años; mientras que parte de la superficie de una empresa está en etapa de plantación, en otra se cosechan los árboles más jóvenes y en otra se voltea a los más añejos para dejar el terreno raso y posteriormente reiniciar el ciclo de plantación.

Los cambios en la estructura agraria y en el uso del suelo que se produjeron en la Argentina en la década de 1990 como resultado de las medidas neoliberales

${ }^{6}$ Información disponible en: http://neamisionesforestal.blogspot.com.ar/p/informacion-tecnica.html

7 Hablamos de "grandes extensiones" tomando como referencia las explotaciones tradicionales de los agricultores misioneros, las cuales tienen en promedio de 20 a 25 hectáreas. En tanto las explotaciones forestales superan las 100 hectáreas. 
fueron funcionales al avance del agronegocio ya que las mismas fueron corroyendo las capacidades de producción de los pequeños y medianos agricultores obligando a muchos a abandonar la producción directa y liquidar sus explotaciones (Chifarelli, 2010).

En este contexto, se registraron procesos migratorios de las áreas rurales a las cabeceras de los distintos departamentos. En efecto, los productores que vendieron sus chacras, se mudaron a los pueblos o ciudades más cercanas, ya sea para establecer un pequeño comercio, convertirse en empleados de ARAUCO o en personal de alguna otra empresa forestal. Muchos otros, sin embargo, no lograron nuevas inserciones ocupacionales, sobreviviendo en base a programas de asistencia, changas, etc.

En el siguiente cuadro es posible observar la variación porcentual de la población rural y urbana en los departamentos de Iguazú, Eldorado y Montecarlo. Con excepción del departamento de Iguazú, en todos los municipios se observa una caída de la población rural y un aumento de la urbana entre los años 1991, 2001 y 2010.

Población rural y urbana en los departamentos del Alto Paraná 1991-2001-2010

\begin{tabular}{ccccccc} 
Municipio & \multicolumn{3}{c}{ Población Rural } & \multicolumn{3}{c}{ Población Urbana } \\
\hline Iguazú & $\mathbf{1 9 9 1}$ & $\mathbf{2 0 0 1}$ & $\mathbf{2 0 1 0}$ & $\mathbf{1 9 9 1}$ & $\mathbf{2 0 0 1}$ & $\mathbf{2 0 1 0}$ \\
\hline Eldorado & $11 \%$ & $6,5 \%$ & $7,6 \%$ & $89 \%$ & $93,5 \%$ & $92,4 \%$ \\
\hline Montecarlo & $29,3 \%$ & $22 \%$ & $20 \%$ & $71 \%$ & $78 \%$ & $80 \%$ \\
\hline
\end{tabular}

Fuente: Cuadro de elaboración propia en base CNPVH 1991, 2001, 2010.

Las chacras abandonadas fueron destinadas a nuevos usos: la forestación. Las plantaciones avanzaron incluso hasta cercar los núcleos urbanos. En varias las entrevistas realizadas surgió la referencia a las escuelas, los barrios y las colonias que han desaparecido: "Es que no hay gente. Pero en la colonia se cierran porque no hay alumnos. Los caminos tampoco se conservan, ya nadie reclama, pero el pino realmente... yo siempre viví del pino pero a nivel social es perjudicial porque no invita a nadie. Es solo para el dueño" (Empresario jubilado. Eldorado, 22 de mayo 2015).

En consecuencia, con las migraciones internas y el avance de las plantaciones forestales sobre territorios antiguamente habitados por productores rurales se produjo una desaparición de colonias rurales, en algunos casos, en otros se observa la disminución de su población, también la pérdida de territorios antes dedicados a la producción de yerba mate, te, tabaco, tung, entre otros. Con estos cambios en el uso del suelo, se observa también que algunas colonias del Alto Paraná que todavía persisten quedan aisladas, rodeadas de plantaciones, proceso que se conoce como arrinconamiento.

$\mathrm{El}$ arrinconamiento y el desplazamiento de las poblaciones locales se inscriben en los procesos de desposesión (Harvey, 2005) comprendidos como los mecanismos de ajuste espacio-temporales que operan en la reasignación de excedentes de capital y trabajo. El proceso incluye la cooptación de las estructuras preexistentes, así como también su confrontación y represión violenta en los casos en que sean incompatibles con las necesidades del capital (Biocca, 2016). 
Ramírez. Un abordaje histórico de la actividad forestal en Misiones: del frente extractivo al agronegocio forestal.

Ciertamente, la desposesión es una de las dinámicas que acompañan la expansión del agronegocio forestal en el Alto Paraná misionero, pero considero que, tal como plantea Biocca (2015), las dinámicas dependen de los lugares y posiciones que toman los diferentes actores envueltos en un proceso que está íntimamente ligado a una historia y una geografía específica. Es decir, el avance del capital no solo opera a través del despojo sino que también puede incluir nuevas formas de explotación, nuevas relaciones de subordinación de los actores preexistentes.

Entre los años 2008 y 2010 se produjo un cambio cualitativo en las empresas forestales relacionado con la mecanización de la actividad sobre todo en la etapa de cosecha. Los empresarios fundamentan la introducción de máquinas cosechadoras en la necesidad de aumentar la productividad por hectárea, reducir tiempos operativos y accidentes de trabajo. Cabe señalar, en ese sentido, que la actividad forestal está considerada entre las más riesgosas del mundo.

La introducción de las máquinas cosechadoras, conocidas como Harvester ${ }^{8}$, repercutió considerablemente en el mercado laboral ya que cada una de esas máquinas reemplaza el trabajo de unas 70 personas por solo dos operarios. A modo de ejemplo, uno de los jóvenes entrevistados que trabaja en una empresa familiar que presta servicios de cosecha para ARAUCO comentó que en su empresa trabajan actualmente alrededor de 25 personas incluido el personal administrativo, pero que hace más de una década, cuando la cosecha no se realizaba mecánicamente, llegaron a emplear alrededor de 100 trabajadores. Así desapareció la figura del motosierrista (obrero forestal que opera con motosierra $)^{9}$ que antiguamente se ocupaba de las tareas de poda, raleo y cosecha: "Antes habia motosierristas, medidor, raleador, existía todo. Ahora no hay más nada" recordó Gustavo, un ex trabajador forestal que vive en Piray km 18 (22 de julio de 2015). Junto con la introducción de las Harvester, se expandió el uso de productos químicos para terminar con las malezas.

Así, se reiteran los relatos que hablan de cómo la tecnología ha desplazado mano de obra. El desempleo generado por los menores requerimientos de mano de obra ha llevado a migraciones internas así también como a otras provincias. En este contexto, cobra relevancia nuevamente la discusión sobre la relación entre capital, tierra y trabajo en estos regímenes de producción contemporáneos.

El arribo de la multinacional ARAUCO, conocida inicialmente con el nombre de Alto Paraná SA (ubicada en Puerto Esperanza) a Misiones en 1996 fue determinante para la evolución del sector forestal en la provincia, pues implicó una transformación significativa en el modelo de producción forestal, incluyendo el abandono de las lógicas que organizaban el modelo de la foresto-industria y su reemplazo por el modelo del agronegocio forestal.

Posteriormente a la adquisición de Alto Paraná, ARAUCO compra también al Citibank las instalaciones de lo que sería el proyecto Celulosa Puerto Piray (CPP) de

8 Para conocer qué es una Harvester ver: https://www.youtube.com/watch? $v=$ soLjGPknv2o

9 A principios de siglo XX el motosierrista sustituyó al hachero, ya que antes de la llegada de la motosierra los árboles se cortaban con hacha y con sierras manuales. 
Celulosa Argentina, proyecto que nunca llegó a concretarse porque la Celulosa quebró a fines de la década de 1980 y el Citibank se quedó con esas acciones y luego se las vendió a ARAUCO. Se trata del lugar en el que hoy se encuentra el aserradero y la fábrica de tableros de media densidad (MDF) en Puerto Piray, inaugurados en 2000 y 2002 respectivamente. Ambos establecimientos se presentan como los más grandes y modernos de la Argentina (ARAUCO, 2013). Para el año 2004 se pone en servicio la línea de productos de pasta fluff en Puerto Esperanza, producto utilizado para la fabricación de pañales descartables y otros de higiene personal ${ }^{10}$. ARAUCO es el único proveedor nacional de este insumo (ARAUCO, 2013).

La empresa multinacional fue ampliando su superficie forestal e integrando eslabones de la cadena de valor a través de sucesivas adquisiciones. En el 2003 compró la división forestal de Petrobras forestal SA (ex Grupo Pérez Companc ${ }^{11}$ ) e incorporó unas 58.000 hectáreas, de las cuales 23.500 ya estaban forestadas. En 2005 ARAUCO adquirió la división de forestal del grupo Louis Dreyfus ${ }^{12}$ en Argentina, que incluye una planta de paneles aglomerados en la localidad de Zárate (pcia. de Buenos Aires) con una producción de $250 \mathrm{~m} 3$ al año y una planta de metanol y resinas en Puerto General San Martín (pcia. de Santa Fe) (ARAUCO, 2013).

Actualmente ARAUCO Argentina es la principal empresa del agronegocio forestal en Misiones (en Argentina, Chile y Uruguay); en esta provincia se concentra el 39\% del área implantada (Gautreau, 2014). En Chile, donde se sitúa la casa matriz desde la década de 1970 se ubica el núcleo más importante de la empresa (con 5 plantas de celulosa y 8 aserraderos). ARAUCO es propietaria de 1 millón de hectáreas de plantaciones en el mundo y operaciones comerciales en Argentina, Australia, Nueva Zelanda, Brasil, Colombia, Europa, Japón, México, Perú, Estados Unidos y Canadá. Según la información proporcionada por los gerentes de esta empresa, en todos los países mencionados los establecimientos industriales son similares y disponen de tecnología moderna. Lo que sí varían son las especies que se cultivan por las condiciones de suelo y climáticas.

ARAUCO está conformada, en rigor, por un conglomerado de empresas a través de las cuales controla buena parte de la cadena de valor forestal, desde la investigación, la instalación de rigurosos ensayos de mejoramiento genético -información que la empresa

${ }^{10}$ Celulosa kraft blanqueada de fibra larga en fardos y en bobinas (fluff); http://www.arauco.cl/ informacion .asp idq $=1231 \&$ parent $=1229 \&$ ca submenu $=3588 \&$ tipo $=3 \&$ idioma $=37$

${ }^{11}$ Perez Companc es uno de los grupos más importantes del empresariado argentino. Actualmente sus negocios apuntan al campo y a la producción de alimentos. Una de sus últimas compras fue una división de la ex Skanska Argentina, dedicada al servicio de pozos petrolíferos y gasíferos en las cuencas más importantes del país. Su principal inversión en la Argentina es la alimenticia Molinos Río de la Plata de la cual posee un $73 \%$ del capital accionario.

${ }^{12}$ Louis Dreyfus Company (LDC) es una compañía global de comercialización de commoditys y procesadores de bienes agrícolas. Sus actividades abarcan toda la cadena de valor. En la Argentina es una de las compañías de mayor envergadura del país, ocupando el quinto lugar entre los exportadores del país. Comercializan oleaginosas, cereales, harina y aceite de soja, biodiesel, arroz, algodón, productos lácteos, fertilizantes, agroquímicos y semillas. Entre sus servicios cuentan con una red de almacenamientos, al igual que "recursos y soluciones financieras" según señalan en su portal http://www.ldcom.com/ar/es/ nosotros/louis-dreyfus-company-en-argentina/ 
Ramírez. Un abordaje histórico de la actividad forestal en Misiones: del frente extractivo al agronegocio forestal.

guarda celosamente-, la producción de este material genético en viveros propios hasta el desarrollo de diversos subproductos con distinto grado de procesamiento industrial. De hecho, en Chile, una empresa del grupo se dedica exclusivamente a desarrollar materiales genéticos y tecnologías para el mejor aprovechamiento de la pulpa y la fibra de la madera (Bioforest). Esta empresa también brinda servicios de biotecnología para sus sedes en Uruguay, Brasil y Argentina.

En Misiones, una de las características más importantes de la multinacional forestal es que realiza su producción por medio de numerosas empresas contratistas. ARAUCO requiere de una serie de empresas prestadoras de servicios en las que terceriza diversas tareas. Esta lógica de contratación de servicios (out-sourcing) no es nueva, Alto Paraná la desarrollaba en la década 1980 y ello tuvo continuidad con la llegada de la empresa ARAUCO, aunque se ha incorporado toda una serie de exigencias, vinculadas a normas internacionales, que la empresa requiere a sus prestadores al momento de contratarlos.

Según la gerencia de operaciones forestales de ARAUCO, en el área forestal prestan servicio alrededor de 70 empresas. Ellos calculan que con la logística (servicios industriales, vigilancia, alimentación, transporte de personal, etc.) la cantidad de empresas vinculadas a ARAUCO arriban al número de 100.

En lo que respecta al servicio de cosecha en Misiones específicamente, son 7 las empresas contratistas. Para con ellas, ARAUCO tiene altos estándares de calidad y regímenes estrictos. Además, la empresa multinacional no realiza contratos con las empresas prestadoras de servicios sino que opera a partir de órdenes de compra que se renuevan anualmente. Esto implica un gran riesgo para las empresas prestadoras, sobre todo para las que realizan trabajos como la cosecha, pues toman créditos para contar con las innovaciones tecnológicas que exige ARAUCO y al mismo tiempo se vuelven cada vez más dependientes de la gran empresa ya que no existe otra a la que puedan ofrecerle servicios en la magnitud que necesitan para mínimamente amortizar las inversiones realizadas.

El arribo de ARAUCO a Misiones aceleró el proceso de concentración empresarial preexistente, ya que se produce una fusión de la empresa Alto Paraná S. A, Pérez Companc y la compra de una estructura de la antigua empresa Celulosa Puerto Piray (CPP). ARAUCO adquirió también tierras que antiguamente pertenecían a Celulosa Argentina destinadas a la forestación en el municipio de Puerto Piray.

En 2010, la empresa inicia el aprovechamiento de residuos de cosecha para la generación de energía a partir de biomasa de origen forestal. Ello ha permitido a la empresa autoabastecerse en todas sus plantas. Además, la empresa dispone de un vivero forestal ubicado en Puerto Bossetti con el objetivo de alcanzar una producción de 3 millones de plantas al año de Eucalipto en contenedores (ARAUCO, 2013).

Cuando ARAUCO adquirió las tierras en Misiones, como parte de sus "operaciones", citando el lenguaje empresarial, no estaba en su horizonte dedicar la mitad de su patrimonio a áreas de reserva natural. Por entonces no regía la ley 26.331 de Presupuestos Mínimos de Protección Ambiental de los bosques nativos, aprobada por el Congreso Nacional en 2009. Ello obligó a ARAUCO a conformar áreas de reserva que, por lo general, no son accesibles para la población local. Los gerentes de esta empresa 
sostienen que es la única forestal en el mundo con tanta extensión dedicada a reservas y algunos empleados afirman convencidos que "solo una empresa grande como ARAUCO es capaz de proteger el medio ambiente", para demostrarlo citan el caso de la Reserva San Jorge, en la que se encuentran especies emblemáticas de la provincia, algunas incluso en peligro de extinción ${ }^{13}$. A través de la publicidad corporativa ARAUCO intenta instalarse como una empresa que cuida el medio ambiente, sin embargo estas tierras están todas concentradas y no fomentan la conectividad del paisaje, esto significa que se produce la pérdida del hábitat de muchos animales y comunidades, y la fragmentación causadas por barreras artificiales, barreras naturales y barreras culturales.

Pero, ARAUCO no sólo compró empresas grandes -Alto Paraná, Celulosa Argentina y del grupo Pérez Companc- sino también aprovechó la "disponibilidad" de tierras resultante del desplazamiento de pequeñas y medianas explotaciones, que fueron especialmente intensos a lo largo de la década de 1990, y de los cuales el CNA de 2002 dio acabada cuenta ${ }^{14}$.

En aproximadamente 10 años (de 1996 a 2006) ARAUCO obtuvo la propiedad de 232.000 hectáreas en Misiones distribuidas en los departamentos de Iguazú, Montecarlo, General Manuel Belgrano, Eldorado, San Pedro, Libertador General San Martín; San Ignacio y Candelaria. Alrededor de 120.000 hectáreas están destinadas a plantaciones con fines productivos. El resto está compuesto por áreas de reservas del monte nativo (selva paranaense).

Sintetizando, sostenemos que el acaparamiento de tierras en manos de ARAUCO no solo es resultado de los volúmenes de capital que controla, a partir de los cuales reestructuró modalidades preexistentes de acceso y control de los recursos, sino también del ejercicio de prácticas simbólicas que refuerzan la presencia de ARAUCO en el territorio. En esta dirección, ARAUCO dispone de un amplio repertorio profesional dedicado a -según afirman los mismos comunicadores de la empresa-, comunicar un "mensaje positivo" hacia la sociedad. La comunicación está basada en distintos mecanismos que van desde acciones de responsabilidad social empresaria, hasta actividades recreativas que buscan involucrar al conjunto de la población local. Las estrategias de la empresa se efectúan en múltiples direcciones y en relación estrecha con los diferentes actores locales en función de reforzar y multiplicar las alianzas.

Al mismo tiempo, la empresa realiza una multiplicidad de aproximaciones a instituciones públicas con la intención de potenciar su comunicación externa y con ello su imagen corporativa con el fin de sostener una presencia activa en el imaginario social. En consonancia con ello se despliegan sistemas de gestión ambiental vinculados a las certificaciones internacionales ${ }^{15}$, de los que el portal institucional de la empresa da acabada cuenta.

${ }^{13}$ Ver video institucional Reserva forestal San Jorge https://www.youtube.com/watch?v=rNguLC9OP8w

${ }^{14}$ Es de suponer que los mismos no se detuvieron con posterioridad a ese registro. Lamentablemente, los datos del último CNA de 2008 adolecen de una serie de problemas de inconsistencia, lo cual no hace aconsejable su uso.

15 "La Certificación Forestal es un instrumento basado en el mercado que persigue aumentar el resguardo y proveer incentivos tanto para productores como consumidores para alcanzar un uso más responsable 
Ramírez. Un abordaje histórico de la actividad forestal en Misiones: del frente extractivo al agronegocio forestal.

Las prácticas de control y vigilancia son un mecanismo fundamental de la empresa para sostener y garantizar su acceso a los recursos. ARAUCO dispone de una dependencia de "protección forestal" cuya responsabilidad es la de evitar "robos, intrusión, la caza furtiva y los incendios" (Jefe de Protección forestal, ARAUCO. Eldorado, 14 de julio 2015). Ese trabajo se realiza con personal de la empresa y eventualmente, la empresa también terceriza algunos servicios específicos. Sin embargo, estas prácticas que están formalizadas dentro de la empresa no son parte de la comunicación corporativa de ARAUCO.

En resumen, el poder de ARAUCO se forja no solo a partir de su titularidad de más de 230.000 hectáreas, sino por una multiplicidad de acciones dirigidas a controlar el acceso a los recursos (materiales y simbólicos). Esa multiplicidad de acciones continuas asegura a la empresa el acceso a los recursos a partir del ejercicio de un "conjunto de poderes" tal como lo definen Ribot y Peluso (2003:153) para evidenciar con ese concepto que las relaciones de poder se crean y recrean constantemente y van más allá del hecho de ejercer derechos de propiedad.

En este sentido, el ejercicio del acaparamiento resulta un proceso continuo-por lo que se precisa redefinir constantemente los mecanismos de acceso, más allá de la propiedad corporativa- que implica dinámicas de desposesión, despojo, incorporación y resistencias que involucran activamente a los actores locales. Estos últimos también discuten, negocian, disputan el acceso a los recursos pues de ellos dependen sus condiciones de subsistencia.

\section{Consideraciones finales}

En las últimas tres décadas, el crecimiento exponencial de las hectáreas con plantaciones forestales y el desarrollo de las industrias de celulosa han convertido a la región del Alto Paraná misionero en un lugar emblemático de la producción forestal. En este sentido, cabe considerar que el agronegocio forestal en Misiones implica procesos de acaparamiento de los recursos, en particular, la tierra, innovaciones tecnológicas y cambios sustantivos en el mercado laboral. Estos procesos expresan el impacto de las lógicas de acumulación globales propias del capitalismo contemporáneo en la región del Alto Paraná misionero.

El avance sistemático de las plantaciones forestales iniciado en la década de 1990, se incrementó de manera sustantiva con la llegada de ARAUCO, produciendo cambios en el paisaje en el Alto Paraná misionero. Si bien en Misiones, la empresa más importante y representativa del agronegocio forestal es la multinacional, la comprensión

de los bosques. Certificación es el proceso de verificación independiente del manejo forestal a un nivel requerido por un estándar dado. La certificación se ha desarrollado en gran medida en respuesta a un consenso internacional, siendo el manejo forestal sustentable la base fundamental de tal proceso. Su éxito depende de los consumidores, inversionistas y otros que proveen de incentivos a los administradores forestales para exigir un manejo forestal sustentable, al preferir la adquisición de productos o la inversión en bosques bien manejados" definición del sitio http://www.uach.cl/proforma/certfor/introducc.htm. Durante años la empresa intentó obtener certificados de gestión forestal sostenible, pero sin modificar su sistema de manejo. Finalmente, decidieron cambiar de certificadora. 
de las dinámicas del agronegocio no debe reducirse al comportamiento de una sola empresa, sino observar su lugar en una configuración compleja en la que intervienen una multiplicidad de actores heterogéneos y desiguales.

Las distintas etapas que se presentan aquí deben pensarse como una continuidad plagada de conflictos y dinámicas en tensión. Esta preocupación por las continuidades históricas fundamenta la necesidad de recuperar las investigaciones y estudios realizados sobre el frente extractivo y el modelo forestal-industrial explicitados en la primera parte del artículo. En este sentido, el frente extractivo, donde la principal actividad económica se basaba en la deforestación, es fundamental para la comprensión de los procesos que se forjaron después.

Una de las principales cuestiones que pretendemos comunicar con este artículo es que el agronegocio, particularmente el agronegocio forestal, no se insertan sobre territorios vacíos sino que existen dinámicas preexistentes que no desaparecen mecánicamente con la llegada de un nuevo modelo productivo. Conocer las características de los procesos previos a través de la reconstrucción histórica permite identificar los procesos y prácticas que persisten, resisten, desaparecen o se transforman.

Por último, y en relación con lo antes expuesto, consideramos de suma importancia investigar la forma en que el agronegocio forestal se inscribe en los territorios en virtud de aportar al análisis y comprensión de los actuales procesos de acumulación capitalista en el agro, las dinámicas y las relaciones sociales resultantes en el escenario contemporáneo. No se trata solo de revisar cómo lo global se desarrolla en un territorio o en un caso específico y/o particular, sino de mostrar cómo los procesos de modernización de la agricultura reconfiguran territorios rurales y agrícolas y redefinen sus relaciones sociales, políticas, económicas y culturales.

\section{Referencias bibliográficas}

Abínzano, R. (1985). Procesos de Integración en una sociedad multiétnica: la provincia argentina de Misiones. Tesis doctoral Departamento de Antropología y Etnología de América. Universidad de Sevilla. Inédita.

Alcaráz, A. (2013). La gestación de una "élite local" durante la explotación yerbatera maderera en el Alto Paraná (1870-1920). Domingo Barthe: un representante paradigmático. Monografía de Maestría, Posadas. UNaM-PPAS. Editorial Universitaria Misiones (en prensa).

Bartolomé, L. (1975). “Colonos, plantadores y agroindustrias. La explotación agrícola familiar en el sudeste de Misiones”. En Desarrollo Económico. núm. 58, vol. 15. Buenos Aires.

Bartolomé, L. (2007). Los colonos de Apóstoles. Estrategias adaptativas y etnicidad en una colonia eslava en Misiones. Posadas: Editorial Universitaria de Misiones.

Bianchi, E. y Szpak, C. (2013). Cadenas globales de producción. Implicancias para el comercio internacional y su gobernanza. Programa de la Cátedra de la OMC. Flacso. 
Ramírez. Un abordaje histórico de la actividad forestal en Misiones: del frente extractivo al agronegocio forestal.

Biocca, M. (2015). The silence of dispossession. The dynmics of agrarian change and the politics of indigenous people in Chaco, Argentina. Dissertation for the degree of philosophiae doctor $(\mathrm{PhD})$. University of Bergen, Norway. Inédita.

Biocca, M. (2016). Más allá de las letras de sangre y fuego. Trayectorias de desposesión en Chaco, Argentina. En: Población \& Sociedad revista de estudios sociales. Vol 23, No 2.

Carámbula, M. (2016). "Imágenes del campo uruguayo en clave de metamorfosis. Cuando las bases estructurales se terminan quebrando". En Revista Ciencias Sociales. N ${ }^{\circ} 36$.

Cerdeillac, J y Piñeiro, D. (2016). "Dossier. Agronegocios y Sociedad rural. Una relación difícil. Presentación". En Revista Ciencias Sociales. N ${ }^{\circ} 36$.

Chifarelli, D. (2010). Acumulación, éxodo y expansión. Un análisis sobre la Agricultura familiar en el norte de Misiones. Buenos Aires: Ediciones INTA.

Ferrero, B. (2006). La selva en disputa. Superposición de cosmografia agraria y ambientalista en la provincia de Misiones. Tesis para optar por el grado de Doctor en Antropología Social. Programa de Posgrado en Antropología Social (PPAS), Universidad nacional de Misiones (UNaM).

Ginzburg, C. (1994). "Indicios. Raíces de un paradigma de inferencias indiciales". En Ginzburg, Mitos, emblemas, indicios. Morfología e historia. Barcelona: Gedisa.

Gómez Lende, S. (2016). "Industria forestal y Acumulación por desposesión en la Argentina: el caso de Alto Paraná S.A. en la Provincia de Misiones". En Campo-Territorio: revista de geografia agraria. Vol. 11, No 22. Pp. 38-68. ISSN 1809-6271.

Gras, C y Hernández, V. (2014). "Agricultura empresarial y globalización: identidades, instituciones y pilares del modelo agribusiness argentino". En Otero, G. (comp). La dieta neoliberal. México: Porrúa.

Gras, C. (2013). Agronegocios en el Cono Sur. Actores sociales, desigualdades y entrelazamientos transregionales. desiguALdades.net. Working Paper Series 50, Berlin: desiguALdades.net International Research Network on Interdependent Inequalities in Latin America.

Harvey, D. (2005). El "nuevo" imperialismo: acumulación por desposesión. Buenos Aires: Clacso.

Heredia, B., Palmeira, M. y Pereira Leite, S (2010). "Sociedade e economia do 'agronegócio' no Brasil”. En Revista Brasileira de Ciências Sociais. VOL. $25 \mathrm{~N}^{\circ} 74$.

Jasinski, A. (2013). Revuelta obrera y masacre en la forestal. Sindicalización y violencia empresaria en tiempos de Yrigoyen. Buenos Aires: Biblios.

Kraustofl, E. (1991). Condiciones de trabajo y calidad de vida de los peones forestales de bosque nativo de Misiones. Depto. De Antropología Social, Universidad Nacional de Misiones. Inédita.

Mastrangelo, A., Scalerandi, V. y Figueroa, M. (2011). "Del recurso natural a la plantación: condiciones de trabajo en la producción forestal del Norte de Misiones". En Mastrangelo y Trpin (comp.) Entre las chacras y plantaciones. Trabajo rural y territorio en producciones que Argentina exporta. Buenos Aires: Ediciones Ciccus.

Ramírez, D. (2017). Etnografiando el agronegocio. Impactos y consecuencias del avance forestal en una comunidad de Piray. Tesis de Doctorado. Programa de Posgrado en Antropología Social (PPAS). Posadas, Misiones. Inédita. 
Scalerandi, V. (2012). La Fábrica en Cabure-í Trabajadores, campesinos y foresto industria en el Noreste de Misiones 1930-1970. Tesis de Maestría en Antropología Social. Universidad Nacional de Misiones (UNaM). Programa de postgrado en Antropología Social (PPAS). Facultad de Humanidades y Ciencias Sociales. Posadas, Misiones.

Schiavoni, G. (2008). "Madereros y agricultores. La constitución de un mercado de tierras de Misiones". En Schiavoni (comp.), Campesinos y agricultores familiares. La cuestión agraria en Misiones a fines del siglo XX. Buenos Aires: Ediciones Ciccus.

Slutzky, D. (2014). Estructura social agraria y agroindustria del Nordeste de la Argentina.

Desde la incorporación a la economía nacional al actual subdesarrollo concentrador y excluyente. Editorial Universitaria.

Valls, M. y M. (2000). Inversiones forestales en la Argentina. Una ley que ofrece un negocio. Comentario crítico de la ley 25.080. La Ley, Tomo 2000-B, Doctrina. Pp 959.

\section{Fuentes}

Censo Nacional de Población, Vivienda y Hábitat (1991; 2001; 2010). http://www. indec.gob.ar/

Censo Nacional Agropecuario (CNA, 1988; 2002). http://www.indec.gob.ar/

"Más despidos en el sector papelero", nota del diario Sol Noticias del 6 de julio de 2014. http://www.elsolnoticias.com.ar/notas/53069-mas-despidos-en-el-sector-papelero.

Nea Misiones Forestal. http://neamisionesforestal.blogspot.com.ar/

Municipalidad de Puerto Piray. www.puertopiray.gob.ar

Louis Dreyfus Company (LDC) http://www.ldcom.com/ar/es/nosotros/louis-dreyfuscompany-en-argentina/

Reserva forestal San Jorge https://www.youtube.com/watch? $v=r N g u L C 9 O P 8 w$

Certificación forestal en chile http://www.uach.cl/proforma/certfor/introducc.htm

ARAUCO. SA https://www.arauco.cl/argentina/?r=1

Reseña histórica de la institución forestal argentina. Bibliotecaria Nilda Fernández. http:// www.agroindustria.gob.ar/new/0-0/forestacion/_archivos/_biblioteca/ifona22.htm

Informe "Evolución del comercio internacional de productos forestales 2010-2013". Peirano, C. (2014), Asociación Forestal Argentina (AFOA), Abril 2014. 
